\title{
G-Proteins Signal Transduction Pathway and $\beta$-Arrestin
}

\author{
Ting Lu* \\ Department of Biological and Chemical Engineering, China
}

*Corresponding author: Ting Lu, Department of Biological and Chemical Engineering, Jichang Road, 10, 617000, Panzhihua, Sichuan, China

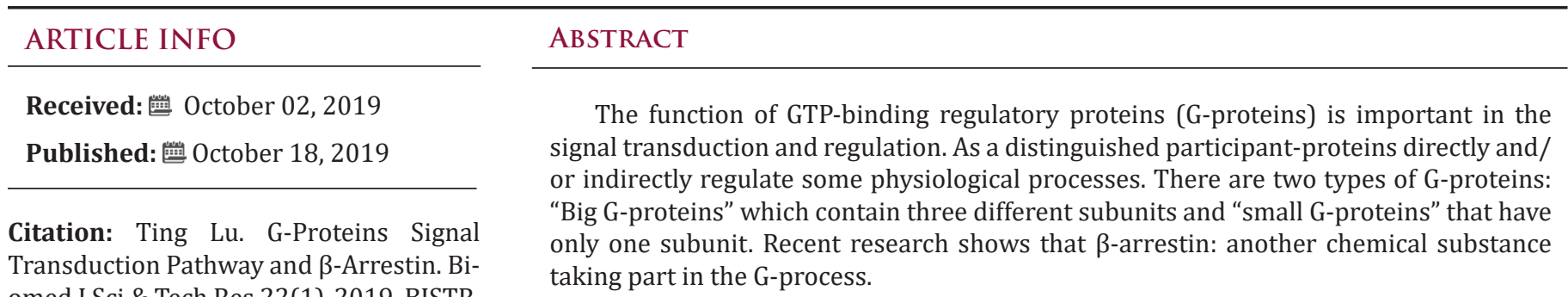
omed J Sci \& Tech Res 22(1)-2019. BJSTR. MS.ID.003707.

Keywords: G-proteins; Signal Transduction; Membrane Receptor; $\beta$-Arrestin

Abbreviations: PE: Phenylephrine; NE: Norepinephrine; IP: Inositol Triphosphate; DAG: Diacylglycerol; PLA: Phosphatidic Acid; GPCR: G Protein-Coupled Receptor; GRKs: G Protein-Coupled Receptor Kinases

\section{Introduction}

The G protein-coupled signaling pathway is an important pathway in cell signaling. Through transmembrane receptors, cells recognize extracellular signals and convert them into intracellular responses. G-protein-coupled transmembrane receptors are formed. The largest family of signals. The G protein binds to GTP and hydrolyzes GTP, so it plays an important role in the intracellular response switch. The large G protein has a trisubunit structure and is divided into four subfamilies according to the functional properties of the $\alpha$ subunit. The activated $\mathrm{G}$ protein signals the downstream effector molecules, such as adenylate cyclase and cAMP, and ultimately completes the entire signal transduction process.

\section{Transmembrane Receptors}

In most signal transduction pathways, extracellular signals are transmitted into the cell through the recognition of transmembrane receptors and the help of transmembrane functional proteins. The extracellular portion of the transmembrane receptor first binds to the signaling molecule. Thereby the receptor is activated, and the conformation of the intracellular portion will change as the receptor is activated. Signals are then signaled, and some transmembrane receptors have the characteristics of ligand-gated ion channels or voltage-gated ion channels. Signals delivered to the cell are activated by second messenger or other receptor recognition [1]. The transmembrane receptor is divided into three domains, consisting of several identical or different subunits, and the transmembrane domain is a channel. Often composed of hydrophobic amino acids. In addition to facilitating the delivery of signaling molecules, it also acts to immobilize the transmembrane portion of the receptor to the phospholipid bilayer. When the signal is passed to the intracellular domain, a series of effector responses need to be activated, including [1]:

a. Activation: G protein, tyrosine kinase, serine. /threonine kinase, lysine phosphatase;

b. Binding ion channel, simultaneously forming a second messenger to activate the subsequent enzyme system, mainly the kinase system.

\section{G-protein Coupled Receptors}

G protein-coupled receptors contain a large class of receptors, such as the AT1 receptor of Ang, the ET-1 receptor of ETA, and the receptors of phenylephrine (PE) and norepinephrine (NE) are G. The protein-coupled receptor binds to the receptor and activates the intracellular GTPase to activate the G protein $\alpha$ subunit and the bg subunit. Ga can further activate downstream phospholipid metabolism and produce a variety of phospholipid 
sources. The second messenger such as cAMP, inositol triphosphate (IP3), diacylglycerol (DAG), phosphatidic acid (PLA), etc., these second messengers further initiate downstream different signal transduction pathways. The structural feature of the $G$ protein-coupled transmembrane receptor is that it has seven transmembrane alpha helices [1]. This is the only structural feature shared by all G-protein coupled receptors and is also a recognition feature of G-protein coupled receptors. In addition to these, according to the homology, $G$ protein-coupled receptors can be classified into three categories: The A receptor family includes rhodopsin/ $\beta$-adrenergic receptors.

Characterized by a series of highly conserved core amino acid residues. In most family A receptors, a disulfide bond connects the E2 and E3 rings. In addition, the C-terminus of the inner side of most receptor cells carries a palmitoylated cysteine residue. The $B$ receptor family includes a calcitonin receptor characterized by a long extracellular $\mathrm{N}$-terminus comprising a series of cysteine residues joined by a disulfide bond into a network. The $\mathrm{C}$ receptor family includes gamma-aminobutyric acid receptors, calcium and glutamate receptors, characterized by a very long extracellular $\mathrm{N}$-terminus, forming an extracellular ligand binding site, which has only one Sulfur bond, and the intracellular third loop is very small [1]. In addition to rhodopsin, the ligand is anchored by a transmembrane helix for those receptors whose ligand is a protein or polypeptide. In addition to the transmembrane domain, a structural portion of the extracellular domain is involved in the binding of the ligand to the receptor. Deproteinization occurs in $G$ protein-coupled receptors. At this time, although the extracellular signal is still stimulated, the signal cannot be transmitted to the cell or transmitted in a weak form. There are two main enzymes involved in the de-enzyme and phosphorylation of the receptor:

a. cAMP-dependent protein kinase;

b. G protein-coupled receptor protein kinase.

De-enzyme is divided into two pathways [1], one is the signal transduction inhibition by the $\mathrm{X}$ protein of the signal chain, and the other is the mutual inhibition between the signal chains, that is, the $\mathrm{X}$ protein in one signal chain regulates another $\mathrm{R}^{*}$ de-enzyme of the signaling pathway. The G protein-coupled receptor (GPCR) functions in the form of a dimer, and the $\beta 2$-type receptor of epinephrine exists as a dimer in the cell.

\section{Effector protein GTPase}

When the signal reaches the cell and is transmitted to the effector, it further causes the effector reaction to generate an intracellular reaction. Among these effector proteins, the most important protein GTPase plays a variety of roles. The most common is the switching action that causes the reaction chain to be open or closed. The GDP enzyme in the form of GTP is in an open state, and the GTPase in the form of GDP is in a closed state. The switching function of the regulatory GTPase is controlled by an ornithine conversion factor, which is an activator protein and a G-nucleus through the GTPase. The glycoside segregation inhibitor acts to function, and the regulatory GTPase acts in a GTPase cycle. Efficient signaling by GTPase depends on the relative ratio between the GTP concentration in the activated state and the GDP concentration in the inactive state [1].

\section{[GTPase].[GTP]/[GTPase].[GDP]=Kdiss.GDP/Kcat.GDP}

\section{Two Types of G-proteins}

One class is Hetrotrimeic G-proteins. Or called a large G protein. These include the alpha subunit (35-55 kDa), the beta subunit (35-36 kDa) and the gamma subunit (8-10 kDa), and the other is the monomeric G protein, or small G protein. The structure and function are similar to the $\alpha$ subunit in the large $G$ protein with a molecular mass of 20-30 kDa. Small G proteins are classified into Ras, Rho and Rab/Ypt subclasses according to different structural homology [2]. Large G proteins can be classified according to the functional properties of different $\alpha$ subunits:

1) Gs subfamily: characterized by its activity inhibited by cholera toxin,

2) Gi subfamily: characterized by inhibitory effects on adenylate cyclase. Self-activity is inhibited by pertussis toxin,

3) Gq subfamily: pertussis toxin and cholera toxin do not regulate member activity of the Gq subfamily,

4) G12 subfamily: its activation requires mediated by thromboxane and thrombin.

The $\beta$ and $\gamma$ subunits generally form a stable complex form. The $\beta$ subunit has a $7 \beta$-fold structure, and after adding the $\gamma$ subunit, it forms a propeller with a structure of 7 leaves and a repeat containing the dipeptide WD [3]. The entire activity of the G protein is only present during the period after it is activated. When GTP binds to the G protein, the $\alpha$ subunit on the G protein is separated from the $\beta \gamma$ complex [3]. At this point in the activated state, the activated $\alpha$ protein directly or indirectly regulates specific physiological and biochemical activities. When the GTPase on the alpha subunit hydrolyzes all of the GTP to GDP, the GTPase is inactivated and the alpha subunit is again bound to the beta gamma complex [4]. The main function of $G$ protein is to couple cell surface receptors and regulate the corresponding physiological and biochemical activities. At the G protein level, signaling through G $\alpha$, GTP can be negatively controlled by RGS and the effector itself. The negative modulation of $\beta \gamma$ complex signal transmission is mediated by light sensing factors [3].

\section{Effector Molecule of G-proteins}

Important effector molecules are phosphatase, adenylate cyclase and cGMP-specific phosphodiesterase. Adenylate cyclase catalyzes the formation of 3'-5' cyclic adenosine monophosphate (cAMP) by ATP, an important second messenger that functions 
by activating protein kinases and is inactivated by degradation by phosphodiesterase. Another major class of effector molecule for $\mathrm{G}$ protein activation are the phosphatase $\mathrm{C}$ and beta subfamilies. To date, at least 11 isomers of mammalian phosphatase $C$ have been known. According to the homology of its sequence, four subfamilies - $\beta, \gamma, \delta, \varepsilon$ have been separated. The most in-depth study is the $\beta$ subfamily and the $\gamma$ subfamily. Phospholipase $C \beta$ is an effector enzyme in the signaling of many $G$ protein-coupled receptors [5]. Phospholipase $\mathrm{C} \gamma$ is involved in a growth factor-dependent signal transduction pathway.

\section{Multiple Functions of Regulatory Factor $\beta$-arrestin}

$\beta$--arrestin ( $\beta$-inhibitor) is an important regulator that began to be emphasized in the late 1990s. It is not a protein-coupled receptor. After phosphorylation of the receptor by G proteincoupled receptor kinases (GRKs), $\beta$-arrestin binds to activate the seven-transmembrane helix receptor (G-protein coupled receptor), thereby regulating their information transmission and internalization. $\beta$--arrestin has the function of binding to a variety of proteins and regulating their functions:

1) $\beta$-arrestin is mainly composed of $\beta$-arrestin 1 and $\beta$-arrestin2 and CXCR4, and different combinations are produced. Different biological effects, thus further expanding the diversity and dexterity of $G$ protein-coupled receptor signal transduction [6],

2) Mdm2 is a negative regulator of $P 53$ protein, and $\beta$-arrestin can bind to Mam2 Mdm2 is brought to the plasma membrane when the $G$ protein receptor is activated. The effect of this action is similar to ARF (Alternative reading frame of INK4a) and MDMX, both of which are proteins that inhibit Mdm2-mediated degradation of P53 protein. ARF can bind to Mdm2, so that Mdm2 in the nucleus is isolated, resulting in an increase in the transactivation of $\mathrm{P} 53$ protein. The combination of $\beta$-arrestin and $\mathrm{Mdm} 2$ also inhibits the action of Mdm2 and P53 proteins to a certain extent and reduces the negative regulation of Mdm2 [6];

3) In addition, $\beta$-arrestin can also act as norepinephrine. The receptor signaling molecule and the inhibitory molecule IkBa of NF-kB molecule prevent IkBa phosphorylation and degradation [6];

4) The study also found that endocytosis of Fz4 (Frizzled4) in human embryonic kidney cells is dependent The Wnt5A protein was added and finally completed under the action of the multifunctional receptor protein $\beta$-arrestin.

$\beta$--arrestin2 plays an unexpected role in the type III signaling transmembrane helix transforming growth factor beta (T $\beta$ RIII) receptor. Similar to the role of $\beta$-arrestin in the $G$ proteincoupled signal transduction pathway, the binding of $\beta$-arrestin to T $\beta$ RIII is also triggered by phosphorylation of the receptor on its pigment plasmid region [7]. However, this phosphorylation is reconciled by the type II TGF-beta receptor (TBRII); whereas T $\beta$ RII is itself a kinase. The association with beta-arrestin leads to the internalization of the receptor [7]. Therefore, the regulatory behavior of $\beta$-arrestin is much broader than it is now believed, and even extends to the entire TGF- $\beta$ receptor family.

\section{Prospects}

In recent years, there have been some new developments and discoveries in the study of $G$ protein signal transduction pathways. For example, the recent role of protein beta inhibitors in information transmission, the researchers observed that opioids and cell membrane opioid receptors can promote the rapid migration of beta inhibitors from the cytoplasm into the nucleus and found beta into the nucleus. Inhibitors can cause chromosome remodeling and induce drug target gene activation [8], which has a long-term regulatory effect on cell function. This discovery opens a new window for further uncovering the mechanism of action of the G pathway. The next study will focus on the following issues:

1) Studying G-protein couples using opioid receptors and chemokine receptors as models. The biological significance and receptor function/structural relationship of the feedback regulation of the receptor signal transduction;

2) The important regulatory proteins (such as $\beta$-arrestin, etc.) in the $G$ protein-coupled receptor signal transduction pathway are the main targets, the system to explore the interaction between cell signal transduction pathways and the network system of cell signal transduction;

3) To study the neural mechanism of opioid addiction and its relationship with learning and memory in molecular, cellular, proteomic and electrophysiological levels [8];

4) Using cell signal transduction as a means to explore the possible mechanisms of the active ingredients and prescriptions of Chinese herbal medicine. With the development and research of this series of questions, the research of signal transduction will take a new step.

\section{Supplementary Material}

No supplementary Material.

\section{Acknowledgement}

The author would like to thank Westerdijkinstitute, KNAW for providing the world-class working environment.

\section{Statement of Ethics}

The authors have no ethical conflicts to disclose.

\section{Disclosure Statement}

The authors have no conflicts of interest to declare.

\section{Funding Sources}

The authors have no funding source to declare. 


\section{References}

1 KRAUSS Gerhard (2003) Biochemistry of Signal Transduction and Regulation.

2 WU Wei Hua, ZHAO Yun Yun (1996) GTP-Binding regulatory proteins in higher plant cell. Acta Botanica Sinica 38(5): 406-413.

3 De Waard M, Liu H, Walker D, Scott VE, Gurnett CA, et al. (1997) Direct binding of G-protein beta gamma complex to voltage-dependent calcium channel. Nature 385(6615): 446-450.

4 Margolskee R F (2002) Molecular mechanisms of bitter and sweet taste transduction. J Bio Chem 277: 1-4.

5 Jin Gui Chen, Sona Pandey, Jirong Huang, José M Alonso, Joseph R Ecker et al. (2004) GCRI acts independently of heterotrimeric G protein in

\section{ISSN: 2574-1241}

DOI: $10.26717 /$ BJSTR.2019.22.003707

Ting Lu. Biomed J Sci \& Tech Res

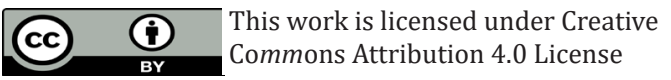

Submission Link: https://biomedres.us/submit-manuscript.php response to brassinosteroids and gibberellic acid in Arabidopsis seed germination. Plant Physiology 135(2): 907-915.

6 FEI Jian (2005) National ‘973’ Research Project - The basis and application research subject of major cell activities: Research proceedings of cell signal transduction. Life Science 4: 286-287.

7 Chen W, Kirkbride KC, How T, Nelson CD, Mo J, et al. (2003) BetaArrestin 2 mediates endocytosis of type III TGF-beta receptor and downregulation of its signaling. Science 301(5638): 1394-1397.

8 Mc Donald PH, Lefkowitz RJ (2001) Beta-Arrestins: new roles in regulating heptahelical receptors' functions. Cell Signal 13(10): 683689. 